\title{
ÉDITORIAL
}

\section{Les avancées en oncologie digestive}

\section{Advances in digestive oncology}

\section{E. Mitry}

Département d'oncologie médicale, institut Curie, hôpital René-Huguenin, 35, rue Dailly, F-92210 Saint-Cloud, France Correspondance : mitry@crh1.org

La richesse de l'actualité en cancérologie digestive au cours des derniers mois nous a semblé justifier la réalisation d'un numéro spécial d'Oncologie.

En effet, avec, par exemple, la disponibilité de nouvelles molécules dites de thérapie ciblée, la présentation des résultats d'études permettant de définir de nouveaux standards thérapeutiques ou d'avoir une meilleure connaissance de la biologie tumorale, la prise en charge des cancers digestifs s'est améliorée mais également parfois complexifiée. Dans ce contexte, nous espérons qu'une synthèse claire et pratique des données récentes les plus importantes, faite par des experts reconnus, vous sera utile.

L'état d'avancement du dépistage organisé du cancer colorectal en France et les modalités futures de ce dépistage, les facteurs prédictifs et pronostiques, les options thérapeutiques en situation métastatique, le traitement de la carcinose péritonéale et le cas particulier de la prise en charge des personnes âgées sont les cinq mises au point concernant le cancer colorectal.

Quatre autres articles sont consacrés au traitement des tumeurs non colorectales : $\mathrm{CHC}$, tumeurs des voies biliaires, tumeurs endocrines digestives et formes non métastatiques des adénocarcinomes pancréatiques.

Un grand merci aux rédacteurs et très bonne lecture. 